\title{
Some Theorems on the Permanent*
}

\author{
R. A. Brualdi and M. Newman
}

(June 9, 1965)

\begin{abstract}
The purpose of this paper is to prove some miscellaneous theorems on the permanent. We define $f(k)$ to be the smallest order of a 0,1 matrix with permanent equal to $k$ and obtain an asymptotic formula for $\log f(k)$. A few theorems concerning the permanent of a circulant matrix are also proved.
\end{abstract}

\section{Introduction}

Let $A=\left[a_{i j}\right]$ be an $n \times n$ complex matrix. The permanent of $A$ is the scalar valued function of $A$ defined by

$$
\operatorname{per}(A)=\Sigma a_{1 i_{1}} a_{2 i_{2}} \ldots a_{n i_{n}}
$$

where the summation extends over all permutations $i_{1}, i_{2}, \ldots, i_{n}$ of $1,2, \ldots . n$. Much of the current interest in the permanent is derived from a conjecture of van der Waerden, and directly or indirectly this conjecture accounts for a great deal of the research that has been done; see e.g., $[1,3,4] .{ }^{1}$ Our purpose here is to prove some miscellaneous theorems on the permanent. In section 2 we define a function on the positive integers by means of the permanent and obtain an asymptotic formula. In section 3 we deal with matrices which are nonnegative circulants. We derive there a congruence and upper bound for the permanent and also a theorem which would be a consequence of the van der Waerden conjecture.

\section{An Asymptotic Formula}

For $k$ a positive integer define $f(k)$ to be the smallest order of a 0,1 matrix with permanent equal to $k$. It of course must be verified that this definition is mean-

*This work was done while the first author was a National Academy of Sciences - National Research Council Postdoctoral Resident Research Associate at the National Bureau of Standards, 1964-1965.

${ }^{1}$ Figures in brackets indicate the literature references at the end of this paper. ingful, and the following example shows this to be so. Define $A_{k}$ to be the $k \times k 0,1$ matrix

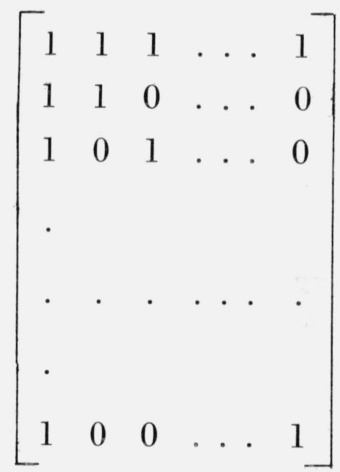

Then it is easily seen that per $\left(A_{k}\right)=k$. The example also shows that $f(k)$ satisfies

$$
f(k) \leqq k, \quad k=1,2, \ldots .
$$

By taking direct sums we find that the function $f$ also satisfies

$$
f(m n) \leqq f(m)+f(n)
$$

where $m$ and $n$ are positive integers. This function $f(k)$ is a very complicated one and how much can be said about it is not clear. However the following theorem shows that $\log f(k)$ is of the same order of magnitude as $\log \log k$. 
THEOREM 2.1. $\log \mathrm{f}(\mathrm{k}) \sim \log \log k$

Proof. Let $A\left(\epsilon_{0}, \epsilon_{1}, \ldots, \epsilon_{n}\right)$ be the following $(n+2)$ $\times(n+2)$ matrix:

$$
\left[\begin{array}{cccccc}
0 & \epsilon_{0} & \epsilon_{1} & \epsilon_{2} & \ldots & \epsilon_{n} \\
1 & 1 & 0 & 0 & \ldots & 0 \\
1 & 1 & 1 & 0 & \ldots & 0 \\
1 & 1 & 1 & 1 & \ldots & 0 \\
. & . & . & . & \ldots & . \\
1 & 1 & 1 & 1 & \ldots & 1
\end{array}\right]
$$

Here we take $\epsilon_{i}=0$ or $1, i=0,1, \ldots, n$. We calculate the permanental minor, $p_{i}$, of $\epsilon_{i}$; that is, the permanent of the $(n+1) \times(n+1)$ matrix obtained from (2.1) by striking out the row and column to which $\epsilon_{i}$ belongs. We have $p_{0}=1, p_{1}=2$, and in general that

$$
p_{i}=2 p_{i-1}, \quad i=1,2, . ., n \text {. }
$$

Hence by induction it follows that

$$
p_{i}=2^{i}, \quad i=0,1, \ldots ., n .
$$

Then by the Laplace expansion for the permanent we obtain

$\operatorname{per}\left(A\left(\epsilon_{0}, \epsilon_{1}, \ldots, \epsilon_{n}\right)\right)=\epsilon_{0}+2 \epsilon_{1}+\ldots+2^{n} \epsilon_{n}$

Now let $k$ be any positive integer and write $k$ in binary form,

$$
k=\epsilon_{0}+2 \epsilon_{1}+\ldots+2^{n} \epsilon_{n}
$$

where $\epsilon_{i}=0$ or 1 for $i=0,1, \ldots, n-1$ and $\epsilon_{n}=1$. Then by the above calculation $A\left(\epsilon_{0}, \epsilon_{1}, \ldots, \epsilon_{n}\right)$ is an $(n+2) \times(n+2) 0,1$ matrix with permanent equal to $k$, so that $f(k) \leqq n+2$. Since $k \geqq 2^{n}$, this implies that

$$
f(k) \leqq \frac{\log k}{\log 2}+2
$$

Thus there is a constant $a>0$ such that

$$
\frac{\log f(k)}{\log \log k}<1+\frac{a}{\log \log k} \text {. }
$$

Now let $r$ be the uniquely determined integer such that

$$
(r-1) !<k \leqq r ! .
$$

Then it is clear that $f(k) \geqq r$. An easy application of Stirling's formula shows that for a suitable constant $b>0$,

$$
r>b \frac{\log k}{\log \log k}
$$

and thus

$$
f(k)>b \frac{\log k}{\log \log k} .
$$

Hence there is a constant $c>0$ such that

$$
\frac{\log f(k)}{\log \log k}>1-c \frac{\log \log \log k}{\log \log k .}
$$

The two inequalities (2.3) and (2.4) imply the theorem upon passage to the limit.

Now let $\mathfrak{A}(n)$ denote the class of all $n \times n 0,1$ matrices. The permanent restricted to $\mathfrak{A}(n)$ is a function mapping $\mathfrak{U}(n)$ into the set of integers $0,1, \ldots, n !$. The following corollary gives some information about its range.

COROLlaRy 2.2. Let $\mathrm{k}$ be an integer with $0 \leqq \mathrm{k} \leqq 2^{\mathrm{n}-1}$. Then there exists a matrix $\mathrm{A}$ in $\mathfrak{Q}(\mathrm{n})$ with permanent equal to $\mathrm{k}$.

Proof. Let $k<2^{n-1}$. Then the binary expansion of $\mathrm{k}$ is

$$
k=\epsilon_{0}+2 \epsilon_{1}+\ldots+2^{n-2} \epsilon_{n-2}
$$

with $\epsilon_{i}=0$ or 1 for $i=0,1, \ldots, n-2$. But then $\mathrm{A}\left(\epsilon_{0}, \epsilon_{1}, \ldots, \epsilon_{n-2}\right)$ is a matrix in $\mathfrak{A}(n)$ with permanent equal to $k$. The $n \times n$ matrix in $\mathfrak{A}(n)$ which is obtained from $A(1,1, \ldots, 1)$ by replacing the 0 in the $(1,1)$ position by a 1 has permanent equal to $2^{n-1}$. This establishes the corollary.

\section{Nonnegative Circulants}

Let $C$ be an $n \times n$ nonnegative circulant matrix so that

$$
C=\left[c_{i j}\right]=c_{0} P^{0}+c_{1} P^{1}+\ldots+c_{n-1} P^{n-1}, P^{0}=I,
$$

where

$$
P=\left[\begin{array}{lllllll}
0 & 1 & 0 & . & . & . & 0 \\
0 & 0 & 1 & . & . & . & 0 \\
. & . & . & . & . & . & . \\
0 & 0 & 0 & . & . & . & 1 \\
1 & 0 & 0 & . & . & . & 0
\end{array}\right] \text {, }
$$

the $n \times n$ full cycle permutation matrix, and $c_{i} \geqq 0$, $i=0,1, \ldots, n-1$. Then the permanent of $C$ is

$$
\operatorname{per}(C)=\sum_{\sigma} c_{1 \sigma(1)} c_{2 \sigma(2)} \ldots c_{n \sigma(n)}
$$

where the summation extends over all permutations $\sigma$ of $1,2, \ldots, n$. Suppose that for $k=0,1, \ldots$, $n-1$ there are precisely $x_{k}$ integers $i$ from $1,2, \ldots$, 
$n$ such that

$$
\sigma(i)-i \equiv k \quad(\bmod n)
$$

Then from the definition of a circulant it follows that

$$
c_{1 \sigma(1)} c_{2 \sigma(2)} \ldots c_{n \sigma(n)}=c_{0}^{x_{0}} c_{1}^{x_{1}} \ldots c_{n-1} x_{n-1}
$$

The $x_{k}$ are nonnegative integers satisfying

$$
x_{0}+x_{1}+\ldots+x_{n-1}=n .
$$

By summing (3.2) over all $i=1,2, \ldots, n$ they also satisfy

$$
0 \cdot x_{0}+1 \cdot x_{1}+\ldots+(n-1) x_{n-1} \equiv 0(\bmod n) .
$$

Hence we may write

$$
\operatorname{per}(C)=\Sigma \mu\left(x_{0}, x_{1}, \ldots, x_{n-1}\right) c_{0}^{x_{0}} c_{1}^{x_{1}} \ldots c_{n-1} x_{n-1}
$$

where the summation extends over all nonnegative integers $x_{0}, x_{1}, \ldots, x_{n-1}$ satisfying (3.3) and (3.4). Here $\mu\left(x_{0}, x_{1}, \ldots, x_{n-1}\right)$ is a nonnegative integer.

THEOREM 3.1. If $\mathrm{C}$ is an $\mathrm{n} \times \mathrm{n}$ nonnegative circulant, then

$$
\text { per }(C) \leqq \frac{\operatorname{tr}\left(C^{n}\right)}{n} \text {, }
$$

where $\operatorname{tr}(\mathrm{X})$ denotes the trace of $\mathrm{X}$.

Proof. We have that

$C^{n}=\sum \frac{n !}{x_{0} ! x_{1} ! \ldots x_{n-1} !} c_{0}^{x} c_{1}^{x_{1}} \ldots c_{n-1} x_{n-1} P^{0 \cdot x_{0}} P^{1 \cdot x_{1}}$

where the summation extends over all nonnegative integers $x_{0}, x_{1}, \ldots, x_{n-1}$ satisfying $x_{0}+x_{1}+\ldots$ $+x_{n-1}=n$. Consider

$$
P^{0 \cdot x_{0}} P^{1} \cdot x_{1} \ldots P^{(n-1) x_{n-1}}=P^{a} .
$$

Now $\operatorname{tr}\left(P^{a}\right)=0$ unless $P^{a}=I$ in which case $\operatorname{tr}\left(P^{a}\right)=n$. But $P^{a}=I$ if and only if

$$
a=0 \cdot x_{0}+1 \cdot x_{1}+\ldots+(n-1) x_{n-1} \equiv 0(\bmod n) .
$$

Hence we may write

$$
\frac{\operatorname{tr}\left(C^{n}\right)}{n}=\sum \frac{n !}{x_{0} ! x_{1} ! \ldots x_{n-1} !} c_{0}^{x_{0}} c_{1}^{x_{1}} \ldots c_{n-1}{ }^{x_{n-1}}
$$

where the summation extends over all nonnegative integers $x_{0}, x_{1}, \ldots ., x_{n-1}$ satisfying (3.3) and (3.4).
But clearly

$$
\begin{array}{r}
\mu\left(x_{0}, x_{1}, \ldots, x_{n-1}\right) \leqq\left(\begin{array}{c}
n \\
x_{0}
\end{array}\right)\left(\begin{array}{c}
n-x_{0} \\
x_{1}
\end{array}\right) \\
\ldots\left(\begin{array}{c}
n-x_{0}-\ldots \cdot-x_{n-1} \\
x_{n}
\end{array}\right)=\frac{n !}{x_{0} ! x_{1} ! \ldots x_{n-1} !} .
\end{array}
$$

Since the $c_{i}$ are nonnegative numbers, inequality (3.6) now follows.

We now assume that the circulant is of prime order.

TheOrem 3.2. Let $\mathrm{p}$ be a prime and $\mathrm{C}=\mathrm{c}_{0} \mathrm{P}^{0}+\mathrm{c}_{1} \mathrm{P}^{1}$ $+\ldots+\mathrm{c}_{\mathrm{p}-1} \mathrm{P}^{\mathrm{p}-1}, a \mathrm{p} \times \mathrm{p}$ circulant with $\mathrm{c}_{0}, \mathrm{c}_{1}, \ldots$, $\mathrm{c}_{\mathrm{p}-1}$ nonnegative integers. Then

$$
\operatorname{per}(C) \equiv c_{0}+c_{1}+\ldots+c_{p-1}(\bmod p) .
$$

Proof. Let $t$ be the permutation of $1,2, \ldots, p$ corresponding to the permutation matrix $P$ given in (3.1); that is, $t(i) \equiv i+1(\bmod p)$. Let $\sigma$ be a permutation distinct from $t^{0}=1, t, \ldots, t^{p-1}$. Suppose $\sigma$ corresponds to the term $c_{0}^{x_{0}} c_{1}^{x_{1}} \ldots c_{p-1} x_{p-1}$ in per $(C)$, so that there are precisely $x_{r} \quad i$ 's with $\sigma(i)-i \equiv r$ $(\bmod p)$. Consider the permutation $t^{k} \sigma t^{-k}$ for $k=0$, $1, \ldots, p-1$. We have that

$$
\begin{aligned}
t^{k} \sigma t^{-k}(i)-i & \equiv t^{k} \sigma(i-k)-i & & (\bmod p) \\
& \equiv \sigma(i-k)+k-i & & (\bmod p) \\
& \equiv \sigma(i-k)-(i-k) & & (\bmod p) .
\end{aligned}
$$

Here $(i-k)$ is to be interpreted as its residue $\bmod p$ in the complete residue system $1,2, \ldots, p$. But then as $i$ varies over the integers $1,2, \ldots, p,(i-k)$ varies over $1,2, \ldots, p$. Hence $\sigma$ and $t^{k} \sigma t^{-k}$ give rise to equal summands $c_{0}^{x_{0}} c_{1}^{x_{1}} \ldots c_{p-1}^{x_{p-1}}$ in per $(C)$. Suppose $t^{k} \sigma t^{-k}=\sigma$ for some $k=1,2, \ldots, p-1$. Then inductively it follows that $t^{j k} \sigma t^{-j k}=\sigma$ for $j=1,2$, .... Thus

$$
t^{j k} \sigma(1)=\sigma t^{j k}(1)
$$

and therefore

$$
\sigma(1)+j k \equiv \sigma(1+j k) \quad(\bmod p)
$$

or

$\sigma(1)-1 \equiv \sigma(1+j k)-(1+j k)(\bmod p), \quad j=1,2, \ldots$.

Here again $1+j k$ is to be interpreted as the residue in the appropriate residue system $\bmod p$. But as $j$ varies over $1,2, \ldots, p$ so does the residue of $1+j k$. For

$$
1+j k \equiv 1+j^{\prime} k(\bmod p), \quad 1 \leqslant j<j^{\prime} \leqslant p,
$$

implies $p \mid\left(j^{\prime}-j\right) k$, the latter being impossible for $p$ a prime. Therefore $\sigma(i)-i \equiv \sigma(1)-1 \equiv a(\bmod p)$, for $i=1,2, \ldots ., p$, and this implies $\sigma=t^{a}$ which is a 
contradiction. Hence the permutations $\sigma, t \sigma t^{-1}$, . . ., $t^{p-1} \sigma t^{-(p-1)}$ are all distinct. Therefore the coefficient of $c_{0}^{x_{0}} c_{1}^{x_{1}} \ldots c_{p-1}^{x_{p-1}}$ in per $(C)$ is congruent to zero modulo $P$. Since this is true for all terms arising from a permutation $\sigma \neq t^{0}, t^{1}, \ldots ., t^{p-1}$, we can conclude that

$$
\begin{aligned}
\operatorname{per}(C) & \equiv c_{0}^{p}+c_{1}^{p}+\ldots+c_{p-1}^{p}(\bmod p) \\
& \equiv c_{0}+c_{1}+\ldots+c_{p-1}(\bmod p)
\end{aligned}
$$

the second congruence following from Fermat's theorem.

Let $\mathfrak{A}(n ; k)$ denote the class of all $n \times n 0,1$ matrices with precisely $k$ l's in each row and column. It has been conjectured by Marshall Hall that

$$
\lim _{n \rightarrow \infty} \min _{A \in \mathfrak{U}(n ; k)} \operatorname{per}(A)=\infty, \quad k \geqq 3 .
$$

This conjecture would be a consequence of the validity of the van der Waerden conjecture which asserts for $A \epsilon \mathfrak{A}(n ; k)$ that

$$
\operatorname{per}(A) \geqq k^{n} \frac{n !}{n^{n}}
$$

Hall's conjecture appears to be about as difficult as that of van der Waerden. The following theorem shows that (3.7) is valid if instead of considering the class $\mathfrak{A}(n ; k)$ we consider the subclass $\mathfrak{C}(n ; k)$ of all $n \times n 0,1$ circulant matrices with $k \geqq 3$ l's in each row and column.

THEOREM 3.3. For $k \geqq 3$,

$$
\lim _{n \rightarrow \infty} \min _{C \in \mathbb{C}(n ; k)} \operatorname{per}(C)=\infty .
$$

Proof. Clearly we need only prove (3.8) for the case $k=3$. Thus let $C \epsilon \subseteq(5 ; 3)$. Without loss of generality we may assume that

$$
C=I+P^{i}+P^{j}, 1 \leqq i<j \leqq n-1,
$$

where $P$ is defined as in (3.1). We define a permutation $\sigma$ of $1,2, \ldots, n$ in the following way:

$$
\begin{array}{lrl}
\sigma(r)=r \quad & r=1, \ldots, j-i \\
\sigma(j-i+r)=j+r & r=1, \ldots, n-j \\
\sigma(n-i+r)=j-i+r, & r=1, \ldots, i .
\end{array}
$$

It is easy to verify that $\sigma$ is indeed a permutation of $1,2, \ldots, n$.

Now

$$
\begin{array}{ll}
\sigma(r)-\mathrm{r} \equiv 0(\bmod n) & r=1, \ldots ., j-i \\
\sigma(j-i-r)-(j-i-r) \equiv i(\bmod n), & r=1, \ldots, n-j \\
\sigma(n-i+r)-(n-i+r) \equiv j(\bmod n), & r=1, \ldots ., i .
\end{array}
$$

Hence the term in per $(C)$ corresponding to the permutation $\sigma$ is equal to

$$
c_{0}^{j-i} c_{i}^{n-j} c_{j}^{i}
$$

which, when $C$ is given by (3.9), is equal to 1 .

Let $t$ be the permutation defined by $t(i) \equiv i+1$ $(\bmod n) . \quad$ As in the proof of the preceding theorem (the primeness was not used in this part) it follows that the permutations $\sigma, t \sigma t^{-1}, \ldots, t^{(n-1)} \sigma t^{-(n-1)}$ all give rise to equal summands $(3.10)$ in per $(C)$. But

$$
t^{k} \sigma t^{-k} \neq \sigma, \quad k=1,2, \ldots, n-1
$$

since the fixed elements of the permutation $\sigma$ are 1 , $2, \ldots ., j-i$ while those of $t^{k} \sigma t^{-k}$ are $\mathbf{1}+k, 2+k, \ldots$, $j-i+k(\bmod n)$. Hence the permutations $\sigma, t \sigma t^{-1}$, ..., $t^{(n-1)} \sigma t^{-(n-1)}$ are all distinct, each contributing 1 to per $(C)$. These along with the three permutations $t^{0}=1, t^{i}, t^{j}$ show that

$$
\operatorname{per}(C) \geqq n+3 \text {. }
$$

Since (3.11) is true for each $C \in \mathbb{C}(n, 3)$, the conclusion (3.8) of the theorem is proved.

In case $n$ equals a prime $p$, inequality (3.11) can be proved by use of theorem 3.2. For by this theorem per $(C) \equiv 3(\bmod p)$, while by [2] per $(C) \geqq 6$. Hence per $(C) \geqq p+3$.

Theorem 3.3 can be generalized to a larger class of matrices in the following way. Let $\mathcal{D}(n ; k)$ denote the class of all $n \times n$ matrices $A$ which are expressible in the form

$A=Q^{i_{1}}+Q^{i_{2}}+\ldots+Q^{i_{k}}$,

$$
0 \leqq i_{1}<\ldots<i_{k} \leqq n-1
$$

for some permutation matrix $Q$ of order $n$. We then have

THEOREM 3.4. For $\mathrm{k} \geqq 3$,

$$
\lim _{n \rightarrow \infty} \min _{A \in \mathfrak{D}(n ; k)} \operatorname{per}(A)=\infty .
$$

Proof. Again we need only prove (3.12) for the case $k=3$. Thus let $A$ be in $\mathcal{D}(n ; 3)$. Without loss of generality we may assume that

$$
A=I+Q^{i}+Q^{j}, \quad 1 \leqq \mathrm{i}<\mathrm{j} \leqq \mathrm{n}-1 .
$$

There exists a permutation matrix $R$ such that

$$
R Q R^{\prime}=P_{n_{1}}+P_{n_{2}} \dot{+} . . \dot{+} P_{n_{r}}
$$

where $P_{n_{\mathrm{s}}}$ is the full cycle permutation matrix of order $n_{s} \geqq 1$. Since the permanent of $A$ is not changed if we permute its rows and columns, we may assume that

$$
Q=P_{n_{1}} \dot{+} P_{n_{2}} \dot{+} \ldots \dot{+} P_{n_{r}} \text {. }
$$

Then

$$
\operatorname{per}\left(I+Q^{i}+Q^{j}\right)=\prod_{s=1}^{r} \operatorname{per}\left(I+P_{n_{s}}^{i}+P_{n_{s}}^{j}\right)
$$


If $n_{s}=1$, then per $\left(I+P_{n_{s}}^{i}+P_{n_{s}}^{j}\right)=3$. If $n_{s}>1$, then either $P_{n_{s}}^{i}$ and $P_{n_{s}}^{j}$ are disjoint permutation matrices or are identical. If they are identical, then

$$
\text { per }\left(I+P_{n_{s}}^{i}+P_{n_{s}}^{j}\right) \geqq 2^{n_{s}}+1 \geqq n_{s}+3 \text {. }
$$

Otherwise we may apply (3.11) to obtain

$$
\operatorname{per}\left(I+P_{n_{s}}^{i}+P_{n_{s}}^{j}\right) \geqq n_{s}+3 \text {. }
$$

By applying these results to (3.13) we are able to conclude that

$$
\begin{aligned}
\operatorname{per}\left(I+Q^{i}+Q^{j}\right) & \geqq n_{1}+\ldots .+n_{r}+3 \\
& =n+3 .
\end{aligned}
$$

From this the theorem follows.

\section{References}

[1] R. A. Brualdi and M. Newman, Inequalities for permanents and permanental minors, Proc. Camb. Phil. Soc. 61, (1965).

[2] M. Hall, Jr., Distinct representatives of subsets. Bull. Amer. Math. Soc. 54, 922-926 (1948).

[3] M. Marcus and M. Newman, Inequalities for the permanent function. Ann. Math. 75, 47-62 (1962).

[4] H. J. Ryser, Combinatorial Mathematics. Carus Math. Monograph, No. 14 (Wiley \& Sons, New York, N.Y., 1962). 